\title{
Enterocolitis following endorectal pull-through procedure in children with Hirschsprung's disease
}

\author{
C. E. Blane ${ }^{1}$, E. Elhalaby ${ }^{2}$, A. G. Coran ${ }^{2}$ \\ ${ }^{1}$ Department of Radiology, Section of Pediatric Radiology, The University of Michigan, Ann Arbor, MI 48109, USA \\ ${ }^{2}$ Department of Surgery, Section of Pediatric Surgery, The University of Michigan, Ann Arbor, MI 48109, USA
}

Received: 13 October 1993/Accepted: 19 November 1993

\begin{abstract}
The enterocolitis following a pull-through in Hirschsprung's disease can be life-threatening and difficult to distinguish clinically from gastroenteritis and post-operative complications. We reviewed retrospectively the abdominal radiographs in our series to identify specific radiographic characteristics of this syndrome in this population. A total of 55 episodes of enterocolitis with an abdominal series at presentation were located in the files of 43 patients following pull-through surgery for Hirschsprung's disease. There were $15 \mathrm{ab}-$ dominal series with other complications of Hirschsprung's disease and surgery (seven cases of small bowel obstruction, one of fistula, one of abscess, six of severe constipation) and 71 surveillance follow-up studies. Radiographs were evaluated for bowel dilatation, airfluid levels, intestinal cut-off sign, spiculation, and pneumatosis. The intestinal cut-off sign with two or more airfluid levels had sensitivity of $68 \%$ and specificity of $83 \%$, with a positive predictive value of 0.71 and overall accuracy of $77 \%$. Our review of enterocolitis following pullthrough in children with Hirschsprung's disease concludes that the constellation of an intestinal cut-off sign and at least two air-fluid levels on the abdominal series strongly suggests the diagnosis.
\end{abstract}

The enterocolitis of Hirschsprung's disease is clinically defined as an explosive watery diarrhea with abdominal distension, often accompanied by fever and vomiting [14]. This serious, occasionally life-threatening complication can occur at any time, both before and after a definitive pull-through procedure [1-4]. One early report suggested a prevalence of $50 \%$ in the population of children with Hirschsprung's disease [1]. Enterocolitis was identified as the major cause of death in children with

Portions of this paper were presented at the Society for Pediatric Radiology meeting in Seattle in May 1993

Correspondence to: C.E.Blane, C.S.Mott Hospital, Pediatric Radiology F 3355/Box 0252, Ann Arbor, MI 48109-0252, USA
Hirschsprung's disease in 1979 [5]. Recent improvements in fluid and electrolyte management, along with hyperalimentation in young children, have improved the mortality rate, but the enterocolitis associated with Hirschsprung's disease remains a serious complication. It can be difficult to differentiate from infectious childhood gastroenteritis and post-operative complications, including small bowel obstruction from adhesions, postoperative abscess and fistula formation, rectal stenosis, and severe constipation. The post-operative enterocolitis associated with Hirschsprung's disease can occur in the immediate post-operative period or later, even more than a year after corrective surgery [1]. Early recognition and initiation of treatment are needed to avoid serious morbidity or even death [1]. Current therapy, in addition to nutritional and fluid balance requirements, includes bowel rest with decompression from above (by nasogastric tube) and below (by rectal tube) as well as antibiotic therapy. This study was designed to identify specific radiographic characteristics of this entity on plain abdominal radiographs.

\section{Patients and methods}

From 1974 to 1992,175 children with Hirschsprung's disease have undergone an endorectal pull-through procedure at our institution. Fifty-seven of these children ( $33 \%$ ) developed at least one episode of enterocolitis (Fig.1). The diagnosis of enterocolitis was assigned by the surgical service on retrospective review of the clinical course of the disease. The radiographic files of 43 of these 57 children were available for review. These 43 children had an initial radiographic abdominal series during 141 visits to the emergency room or surgical clinic. Fifty-five of the 141 radiographic abdominal series were obtained prior to any specific treatment in 31 of the 43 patients. In 12 of the 43 patients with an episode of enterocolitis there were no abdominal series prior to initiation of treatment. Fifteen of the 141 abdominal series in the 43 children were performed for other complications of Hirschsprung's disease and the surgery (seven cases of small bowel obstruction, one of fistula, one of abscess, six of severe constipation), and 71 were surveillance follow-up studies. All the plain radiographs were reviewed retrospectively, but without the reviewer having knowledge of the clinical problem at the time of the 


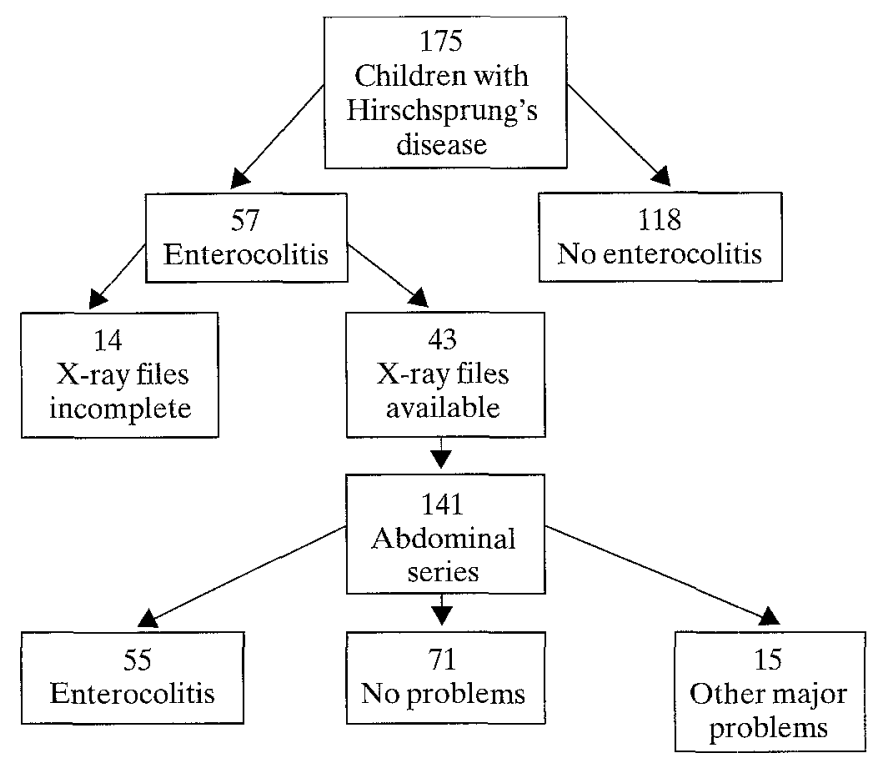

Fig. 1. The selection of abdominal series used in this study

study. A subjective grading scale of bowel dilatation from 0 (no dilatation) to 3 (marked dilatation) was devised. The number of airfluid levels was recorded. An intestinal cut-off sign in the left lower quadrant was defined as gaseous distention of a bowel loop along the left flank with abrupt termination within the left pelvis. If pre- sent, this intestinal cut-off sign was noted. A search of the plain radiographs was made for evidence of spiculation and pneumatosis. The time of onset of the enterocolitis in relation to the pull-through was documented.

\section{Results}

Neither spiculation nor pneumatosis was recognized on any plain abdominal radiograph in this series of children. The intestinal cut-off sign had a sensitivity of $73 \%$ and specificity of $76 \%$ [6]. It was seen in 40 of the 55 episodes of enterocolitis $(73 \%)$, and in 21 of the other $86(24 \%)$ abdominal series (including 2 children with small bowel obstruction from adhesions, 1 with severe constipation, and 18 surveillance studies). The positive predictive value was 0.655 and the overall accuracy was $74 \%$. An intestinal cut-off sign with two or more air-fluid levels decreased the sensitivity to $68 \%$, but increased the specificity to $83 \%$, the positive predictive value to 0.71 , and the overall accuracy to $77 \%$. An intestinal cut-off sign with two or more air-fluid levels and grade 2 or 3 dilatation of bowel had sensitivity of $68 \%$, specificity of $83 \%$, a positive predictive value of 0.71 , and overall accuracy of $77 \%$. Figure 2 illustrates the radiographic findings in a patient with enterocolitis. Figure 3 has an abnormal appearance, but is one of the 15 falsely negative series en-
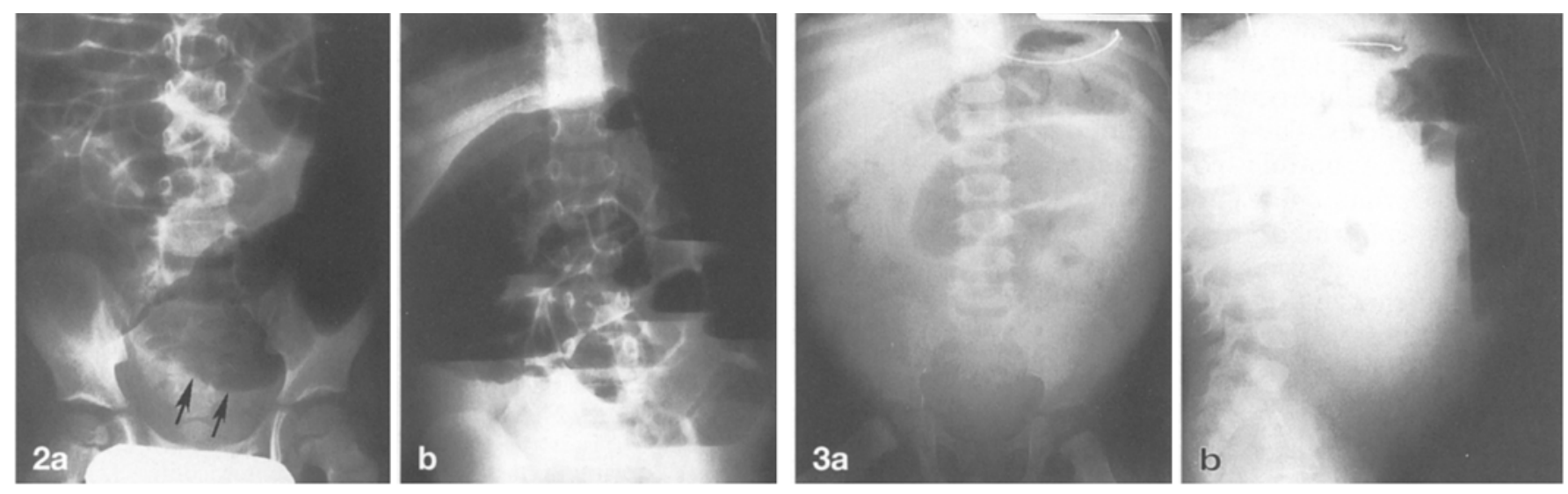
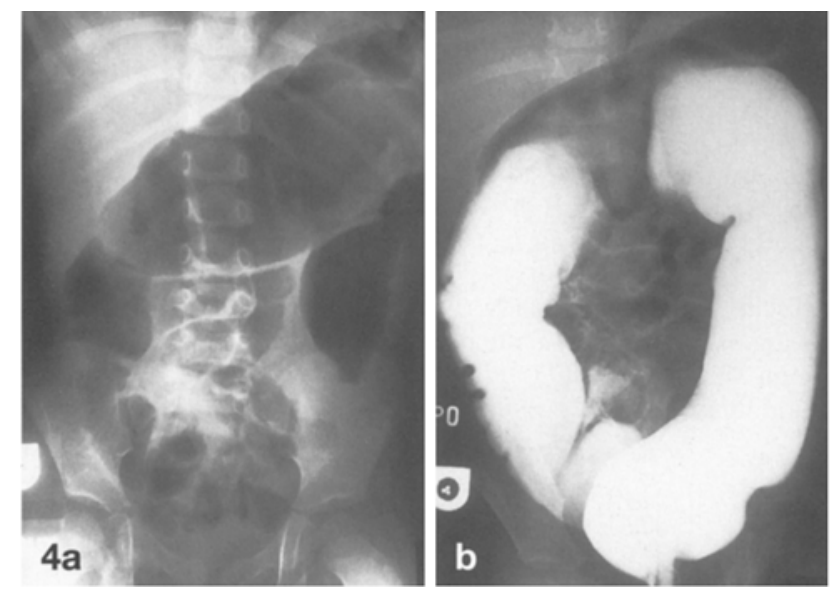

Fig. 4a,b. A 2-year-old girl who had had a pull-through in the neonatal period was hospitalized for enterocolitis. The plain radiograph a taken at the time of hospitalization reveals an intestinal cut-off sign and dilated loops of bowel. The barium enema $\mathbf{b}$ shows no obstruction

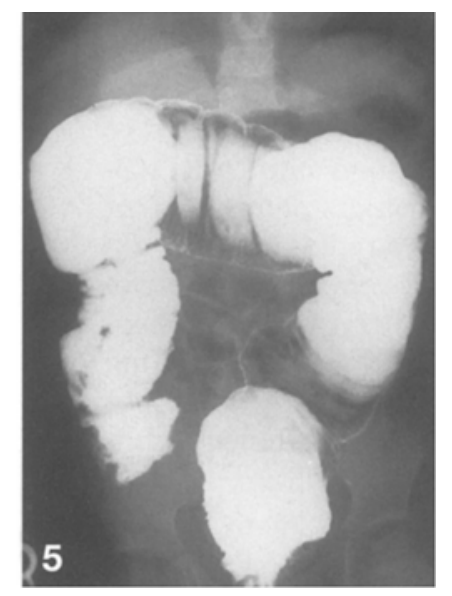

Fig. 2a,b. A 4-year-old boy, who had had a pull-through for Hirschsprung's disease at 2 months of age presented with explosive bloody diarrhea clinically diagnosed as enterocolitis. The intestinal cut-off sign in the left pelvis (arrows) on the supine view a and airfluid levels on the upright view $\mathbf{b}$ can be seen. He also had a dilated bowel

Fig. 3a,b. A 1-year-old boy who had had a pull-through 2 months previously was admitted with the clinical diagnosis of enterocolitis. There is no intestinal cut-off sign. Dilated loops of bowel on the supine view a with air-fluid levels on the cross-table lateral view $\mathbf{b}$ are evident

Fig.5. A $2 \frac{1}{2}$-year-old girl was well at the time of this enema. She had had an episode of enterocolitis 7 months prior to the enema and a pull-through more than 2 years previously. Spiculation is seen at the rectum, caecum and ascending colon 
countered using the above criteria since there is no intestinal cut-off sign. The enterocolitis attacks occurred from 1 month to 176 months following endorectal pullthrough, with a mean of 29 months and a median of 18 months.

\section{Discussion}

One-third of our series of children with pull-through procedures for Hirschsprung's disease in the period 1974-1992 developed enterocolitis following corrective surgery. The mean and median time of presentation were both more than 18 months after successful operation. This significant complication is well recognized in the surgical literature $[1,3-5]$, but has received little attention in the radiological literature. Early papers on enterocolitis in the child with Hirschsprung's disease suggested that this complication was related to a variable degree of mechanical obstruction $[1,3]$. It was postulated that chronic distension as a result of obstruction resulted in impaired vascularity leading to ischemia [5]. Specific pathogens were not identified, but mucosal damage from the ischemia was thought to permit the passage of bacteria and antigens through the intestinal wall, thus establishing hypersensitivity reactions. The theory of obstruction does not explain post-operative enterocolitis in children undergoing successful resection of the aganglionic segment [3]. In our series, one of the patients with post-operative enterocolitis had a barium enema at the time of diagnosis. The plain abdominal radiograph revealed dilated bowel and an intestinal cut-off sign (Fig. 4a). Barium enema at the time of diagnosis of enterocolitis confirmed that there was no obstruction (Fig. 3 a). It was also noted that those children with the most significant pre-operative obstruction were not necessarily those that developed pre-operative enterocolitis [3].

The possibility of a Shwartzman reaction within the mucosa has been suggested [3]. Berry described extensive loss of mucosa, demonstrated histologically, after enterocolitis both in a child with this complication preoperatively and in another who had undergone surgical resection of the aganglionic segment and a successful pull-through [4]. He also suggested that the changes were more severe and persisted longer than clinically suspected [4]. This persistence of histologic changes after clinical resolution of enterocolitis may explain the extensive spiculation seen on the barium enema in a child in our series who was previously admitted with the diagnosis of enterocolitis, but who at the time of the enema was thought to be clinically well (Fig.5). More recently, Branski and Lebenthal have suggested that some chil- dren with enterocolitis following successful surgery for Hirschsprung's disease have a severe disaccharidase deficiency and sugar intolerance [2]. They also found persistent mucosal changes long after surgery [2].

The intestinal cut-off sign was initially described as a colon cut-off sign in acute pancreatitis, with dilatation of the ascending colon and hepatic flexure and abrupt cutoff of the air column in the transverse or descending colon [7]. This finding has been observed in other conditions [8]. Because several of our patients with total colonic Hirschsprung's disease have had resection of the entire colon, and because of the difficulty in differentiating small from large bowel in some younger children, we have defined the distension of a loop of bowel along the left flank with abrupt termination in the pelvis as an intestinal cut-off sign (Fig. 2). This finding, with at least two air-fluid levels, is suggestive of the diagnosis of enterocolitis in the patient with Hirschsprung's disease after pull-through procedure. The additional finding of dilated bowel does not significantly change the specificity.

Enterocolitis following endorectal pull-through in the patient with Hirschsprung's disease is serious and can be seen years after successful pull-through surgery. The plain radiograph findings of two or more air-fluid levels and an intestinal cut-off sign in the left lower quadrant strongly suggest the diagnosis. Enterocolitis following successful surgery for Hirschsprung's disease is an important diagnosis that radiologists must consider in the evaluation of abdominal radiographs in affected children.

\section{References}

1. Bill AH Jr, Chapman ND (1962) The enterocolitis of Hirschsprung's disease. Am J Surg 103: 70-74

2. Branski D, Lebenthal E (1979) Small intestinal changes in enterocolitis complicating Hirschsprung's disease. J Clin Gastroenterol 1: 237-240

3. Fraser GC, Berry C (1967) Mortality in neonatal Hirschsprung's disease: with particular reference to enterocolitis. J Pediatr Surg 2: 205-211

4. Berry CL (1969) Persistent changes in the large bowel following the enterocolitis associated with Hirschsprung's disease. J Pathol 97: 731-732

5. Gryboski JD (1979) The enterocolitis of Hirschsprung's disease. J Clin Gastroenterol 1:248-249

6. Brismar J, Jacobsson B (1990) Definition of terms used to judge the efficacy of diagnostic tests: a graphic approach. AJR 155: 621623

7. Brascho DJ, Reynolds TN, Zanca P (1962) The radiographic "colon cut-off sign" in acute pancreatitis. Radiology 79: 763-768

8. Eaton SB Jr, Ferrucci JT Jr (1973) Radiology of the Pancreas and Duodenum, Saunders, Philadelphia, pp 28-34 